\title{
Breakpoint Tuning in DCT-Based Nonlinear Layered Video Codecs
}

\author{
Pedro Cuenca \\ Departamento de Informática, Escuela Politécnica Superior de Albacete, Universidad de Castilla-La Mancha, \\ Campus Universitario, 02071 Albacete, Spain \\ Email: pedro.cuenca@uclm.es

\section{Luis Orozco-Barbosa} \\ Departamento de Informática, Escuela Politécnica Superior de Albacete, Universidad de Castilla-La Mancha, \\ Campus Universitario, 02071 Albacete, Spain \\ Email:luis.orozco@uclm.es

\section{Francisco Delicado} \\ Departamento de Informática, Escuela Politécnica Superior de Albacete, Universidad de Castilla-La Mancha, \\ Campus Universitario, 02071 Albacete, Spain \\ Email: franciscomanue.delicado@uclm.es \\ Antonio Garrido \\ Departamento de Informática, Escuela Politécnica Superior de Albacete, Universidad de Castilla-La Mancha, \\ Campus Universitario, 02071 Albacete, Spain \\ Email: antonio.garrido@uclm.es
}

Received 31 August 2003; Revised 2 February 2004

\begin{abstract}
Many studies have been conducted to evaluate the benefits of using layered video coding schemes as a means to improve the robustness of video communications systems. In this paper, we study a frame-aware nonlinear layering scheme for the transport of a DCT-based video over packet-switched networks. This scheme takes into account the relevance of the different elements of the video sequence composing the encoded video sequence. Throughout a detailed study over a large set of video streams, we show that by properly tuning the encoding parameters, it is feasible to gracefully degrade or even maintain the video quality while reducing the amount of data representing the video sequence. We then provide the major guidelines to properly tune up the encoding parameters allowing us to set the basis towards the development of more robust video communications systems.
\end{abstract}

Keywords and phrases: DCT, nonlinear layering video coding, video communications, video quality.

\section{INTRODUCTION}

Recent developments in the areas of video coding and compression techniques are enabling the deployment of computer-based video communications systems. In a video communications system, it is essential to count with a reliable support that is able to guarantee the timely and reliable transport of a video stream. The video process must however incorporate the essential elements to react to potential changes in the service provided by the network.

The fact that most video coding schemes use compression techniques makes video communications applications very vulnerable to losses. In the absence of any error control mechanism, the loss of video data causes the loss of information up to the next resynchronization point (e.g., slice head- ers). In other words, a packet loss will translate in the loss of a partial video slice, where a slice is a full-length row of the image (a whole strip on the screen). This is due to the fact that the slice headers are used as the basic resynchronization points in the video signal. The macroblocks forming a slice contain information coded differentially with respect to precedent macroblocks. More specifically, when a macroblock is lost, all the macroblocks that follow up to the end of the current slice cannot be decoded. This is referred to as spatial loss propagation (Figure 1). Obviously, the amount of data actually lost will depend on the relative position of the lost information within the slice. On the other hand, due to the predictive nature of most video coding schemes, when losses occur in a reference picture, the impairment will propagate until the next intracoded picture is received. 


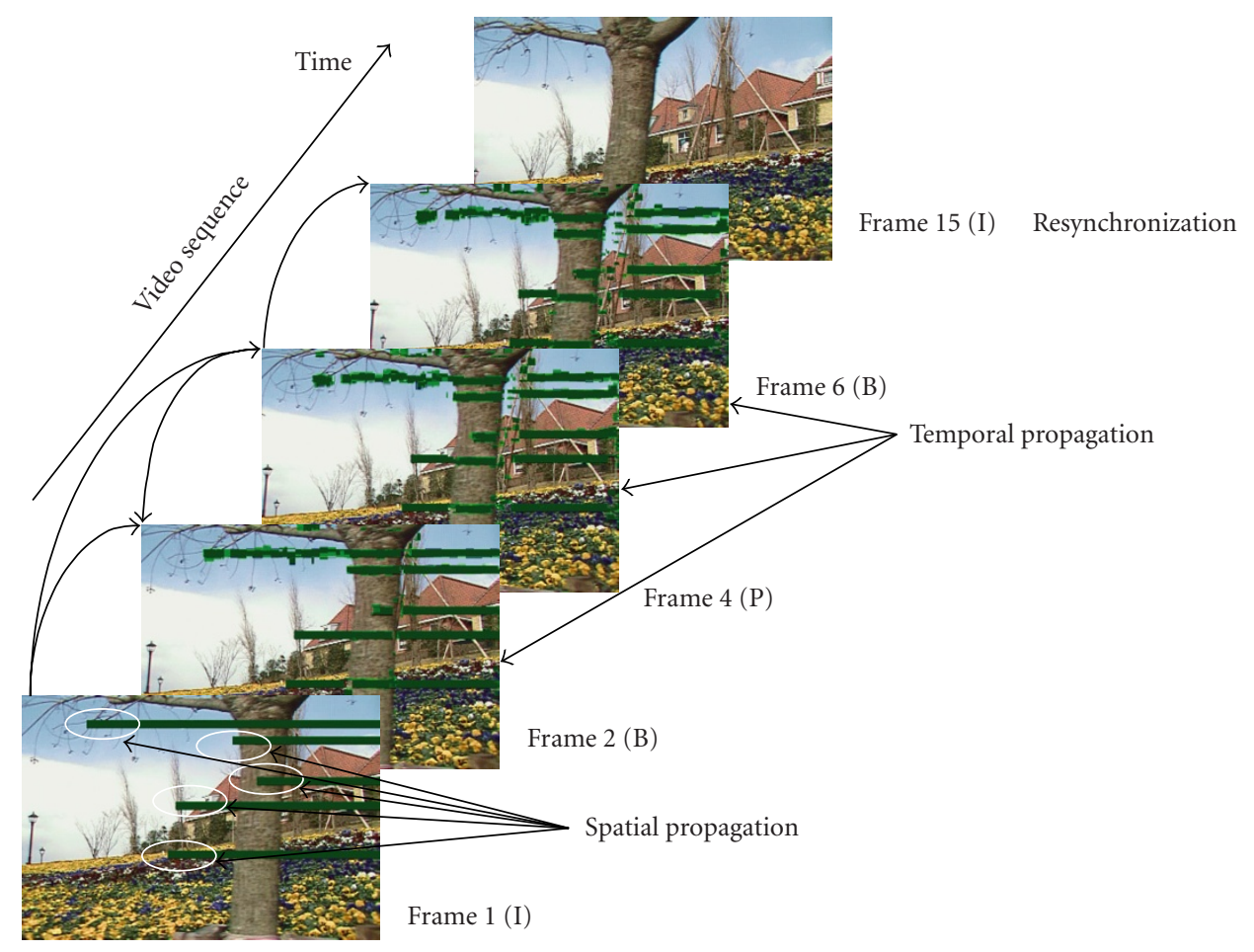

FIgURE 1: Spatial and temporal propagation phenomena.

That is to say, the impairment will propagate through the whole group of pictures (GOP) associated to the impaired reference frame. This effect is known as temporal loss propagation (Figure 1). These phenomena will affect the quality of the video signal, and without adequate controls to locate the propagation of the impairments, the quality of the services (QoSs) may fall below acceptable levels [1].

Most techniques used for the reliable transfer of video over communications networks can be classified into two classes [2]: error-resilient techniques and regeneration techniques. The former are implemented in the codecs as well as in the switching elements of the networks, while the latter are implemented by the decoder by making use of redundancies present in the encoded stream to regenerate the missing pieces of information. Both techniques can be combined to develop structured error-resilient video communications systems [3].

The regeneration techniques can be reinforced using layered encoding. Under a layered encoding scheme, the most relevant elements of the video sequence are included in a base layer, while less relevant pieces of information are put into a second level, also denominated enhancement layer. According to their relevance, the base layer receives a highpriority treatment while the other layer is delegated to a second plane. The base layer provides by itself a minimum acceptable quality video image. One of the main advantages is that this type of encoding scheme can be applied to all discrete cosine transform (DCT)-based encoding scheme, such as H.261, H.263, MPEG-1, MPEG-2, MPEG-4, H.264, among others $[4,5,6,7,8,9,10,11,12,13,14,15,16,17]$.
Video encoders incorporating these features could be used to develop QoS-aware video communications systems. For instance, such video encoders could adapt its encoding parameters in response to a congestion signal from the network. In this way, the encoders could temporally reduce the video generation rate while maintaining a minimum acceptable video quality. However, the effectiveness of such schemes will highly depend on the way the encoding parameters are set up.

In this paper, we present a frame-aware nonlinear layering scheme particularly designed for encoding video sequence making use of a DCT-based scheme. The scheme is based on properly setting up the encoding parameters aiming to improve the quality of the video images while reducing the bit rate of the video sources. Experimental results using a representative set of seven video clips show the feasibility of the proposed scheme. Furthermore, the scheme can prove particularly useful in supporting video communications systems over packet-switched networks. The paper is organized as follows. In Section 2, the principles of operation of DCT-based layered video codecs are described. Experimental results are provided to illustrate the operation and performance issues in terms of overhead, video source bit rates, and image quality. In Section 3, we describe a novel nonlinear encoding scheme. Numerical results show that the proposed scheme outperforms previously reported encoding schemes by considerably reducing the source video rate while guaranteeing a graceful video quality degradation. Section 4 concludes the paper. 




Figure 2: Implementation of DCT-based layered video codecs.

\section{DCT-BASED LAYERED VIDEO CODECS}

Various DCT-based layered video coding schemes have been proposed in the literature in order to improve the robustness of video communications applications $[3,18,19,20,21]$. The main idea is based on the same principle: the insensibility of the human visual system to high-frequency components of the video signal. Under these schemes, the low-frequency DCT coefficients together with other relevant information are transmitted at a high-priority (HP) level, also denominated base layer. High-frequency DCT coefficients and other less relevant information are then transmitted at a lowerpriority (LP) level, also denominated enhancement layer. If parts of the enhancement layer are lost, they are simply replaced by zeros and the image reconstructed using the base layer and the dummy enhancement layer-though somewhat distorted - may be acceptable.

This scheme can be easily adapted to transmission networks which support different QoS levels as ATM, Hiperlan/2, 802.11a/b networks or to network protocols which support different QoS levels, such as IntServ, Diffserv, and MPLS [22, 23, 24, 25, 26, 27, 28]. By using these layered video codecs, a correct transmission of the most important information of the video signal can be somehow guaranteed $[29,30]$. Furthermore, the base layer can be designed so that it can provide by itself a minimum acceptable image quality in situations in which the enhancement layer is completely lost. In these cases, temporary reduction of video quality target and graceful quality degradation is obtained.

\subsection{Implementation issues}

The principles of implementation of DCT-based layered video codecs can be explained as follows (Figure 2). A bitstream break point is first defined, denominated from now on simply as breakpoint. The breakpoint defines the number of DCT coefficients different from zero in a block (apart from the DC coefficient in the case of intra-macroblocks) to be placed in the base layer, while the remaining DCT coefficients are to be placed in the enhancement layer. The base layer contains all the headers and all the control information at the macroblock level, such as motion vectors, macroblock type, motion type, relative address of the macroblock in the slice, as well as the DCT coefficient (that is, the coefficient of continuous DC), of each block encoded as intra. The base layer will also contain all the DCT coefficients different from zero, if any, up to the point indicated by the breakpoint. The remaining DCT coefficients different from zero, up to the end of block (EOB) will make part of the enhancement layer. In the case that the number of coefficients different from zero in one block is lower than the number specified by the breakpoint, an EOB marker is inserted at the end of the base layer, leaving empty the enhancement layer. Sequence headers, GOP, picture, slice, and end of sequence make part of the enhancement layer. The insertion of these headers in the enhancement layer is the only extra overhead added to the bitstream. In the case of errors or losses, this information will be used to resynchronize the two partitions.

The decoding process can be described as follows. The decoder starts by extracting the headers of the two partitions. Upon receiving a block, the DCT coefficients up to the breakpoint are decoded and placed in the base layer. When the breakpoint is reached, if an EOB has not been found, the following DCT coefficients are decoded in the enhancement layer until the EOB is reached. At this point, the decoder will restart the decoding process for a new block. In the case when the decoder finds an EOB without having previously identified a breakpoint, it assumes that there is no enhancement 
layer for the current block. It then starts the decoding process of the base layer for the new incoming block.

In the case of error or loss of information in the enhancement layer, the decoder uses only the information from the base layer to reconstruct the video signal until a resynchronization point between the two layers is found. The resynchronization between both layers is done at the header-code level and it can be achieved by comparing the headers included in the two splits. Synchronization is achieved when the headers included in the two partitions coincide. The macroblocks decoded using only the base layer will present lower quality than those decoded using both layers. If the error or loss of information is produced in the base layer, then the decoder must discard all the information received until finding the next header code.

The implementation of a layered video codec is obtained at the expense of introducing some overhead needed for the mechanism to operate. Therefore, various implementation issues must be considered when designing layered video codecs, such as the amount of overhead required to implement it, the definition of the breakpoint used when splitting the encoded video bitstream into the base and enhancement layers, and the ability of assigning different priorities to the underlying network mechanisms.

Various DCT-based layered video coding studies have already been reported in the literature [3,20,21]. In [20], the overhead associated to the layered coding scheme presented therein is 20\%; this high overhead is due to code words added to the two substreams. In [21], a more efficient implementation is described. However, the introduced overhead remains high. For instance, an overhead of $9 \%$ is introduced when applying the proposed scheme to the Flower Garden video stream. This is due to the fact that the scheme requires including all the headers for each slice for both substreams. The scheme proposed in [3] offers advantages over similar schemes proposed in [20,21]. In the scheme proposed in [3], the overhead introduced is $1.8 \%$ for the same sequence. This has been achieved by including all headers at the beginning of the sequence, but not at each slice since only the slice headers are needed to maintain proper synchronization.

\subsection{A case study: the MPEG-2 video standard}

The MPEG-2 video coding standard developed by ISO/IEC $[5,6,7]$ defines a generic video coding method that addresses a wide range of applications, bit rates, resolutions, qualities, and services. These different requirements have been integrated into a single syntax, which facilitates the bitstream interchange among different applications. The basic requirements of MPEG-2 video coding are a high compression ratio with good image quality and the support of a number of optional features, such as random access, fast search, reverse playback, and so forth.

To achieve a high compression ratio, the temporal and spatial redundancies present in raw video sequences must be removed as much as possible. The MPEG-2 video coding standard is based upon a hybrid coding structure of temporal and spatial processing. In terms of spatial processing, MPEG2 defines a DCT. In terms of temporal processing, MPEG-
2 defines three main frame types: I- (intra), P- (predictive), and B- (bidirectionally predictive) frames. The I-frames are coded without reference to any other frame. They provide the access points to the coded bitstream where the decoding process can begin. The P-frames are predictive coded frames; their references are the previously coded I- or P-frames. The $\mathrm{B}$-frames are bidirectionally predictive coded pictures. They have two references: one from the past and a second from the future. The organization of the three types of frames is very flexible so as to support a wide range of applications. The three types of frames vary with respect to their relevance to the reconstruction of the video signal by the receiver. Iframes are more important than P- and B-frames, since the information contained in an I-frame is used as reference to decode the $\mathrm{P}$ - and $\mathrm{B}$-frames. If no provisions are taken during the transmission of an MPEG-2-encoded video sequence, errors or losses of part or all of the data contained in an Iframe will affect the decoding process of all $\mathrm{P}, \mathrm{B}$ depending on it. Therefore, the performance of communication networks when handling video applications will greatly depend on the way the video signals are encoded as well as on the use of proper control mechanisms used in the transport of the video stream.

We start by considering the performance evaluation of a DCT-MPEG-2 layer video codec when defining a constant breakpoint for the whole video sequence as previously described. Under this scheme, a digital MPEG-2 video stream is encoded into two sub-bitstreams. This partitioning is done by taking into account the relevance of the different pieces of information of the MPEG-2 bitstream (fully compatible with the MPEG-2 data-partitioning scalable profile specifications $[5,6,7])$. In layered video communications, the base layer and the enhancement layer need to preserve the structure of the base layer stream. In the case of MPEG-2 standard, this means complete MPEG-2 transport stream (TS) structuring in both layers (see Figure 3). The MPEG-2 TS is intended for multi-program applications such as broadcasting and for non-error-free environments. All the MPEG-2 TS packets are given extra error protection using methods such as Reed-Solomon encoding [5, 6, 7]. Prioritization of the base layer over the enhancement layer in an MPEG-2 scalable data-partitioning profile using MPEG-2 TS specifications can be done very easily using the transport-priority field (TS header) and packetized elementary stream (PES)priority field (PES header) $[5,6,7]$.

In order to set the basis towards the definition of a nonlinear layered encoding scheme, we carried an exhaustive study by applying the DCT-MPEG-2 layer video codec scheme to seven different video sequences, each one encoded using five different $Q$-factor values.

Table 1 shows the video sequences characteristics: mean bit rates, frame rates, and the share of bit rate used for all DCT coefficients (DCT-bits) and for the rest of the coded data (Hdr-bits): sequence, picture, and macroblock header data. The picture size of the all video sequences is $720 \times 480$ (NTSC CCIR 601). The GOP pattern was set to $N=12$, $M=3$, in MPEG-2 terminology [5, 6, 7]. The video streams were encoded several times using different breakpoint values. 




FIGURE 3: MPEG-2 transport stream generation from layered video frames.

Figure 4 shows the results obtained for the Ayersroc, Hook, and Table Tennis video sequences. Similar results were obtained when applying this scheme to the other four video sequences.

Figure 4 shows that the enhancement layer (LP) can represent a significant portion of the overall bandwidth requirements. It can also be observed that by varying the breakpoint, we can vary the amount of video data to be included in each layer. For instance, in the case of the Ayersroc, Hook, and Table Tennis sequences using a breakpoint of five, the enhancement layer accounts approximately for $33 \%, 22 \%, 15 \%, 10 \%$, $8 \%$ (Ayersroc); 39\%, 28\%, 17\%, 9\%, 7\% (Hook); and 52\%, $42 \%, 29 \%, 19 \%, 11 \%$ (Table Tennis) of the total video data when encoded with a $Q$-factor set to $8,12,20,28$, and 40 , respectively.

In the following, we will present a quantitative assessment of our video quality results using the moving pictures quality metric (MPQM) [31]. MPQM has been proved to behave consistently with human judgments according to the quality scale that is often used for subjective testing in the engineering community (see Table 2) [32,33]. The metric has been developed based on a spatio-temporal model of the human vision system. Therefore, the metric overcomes the lack of correlation of traditional metrics, such as PSNR among others, with human perception. MPQM is based on the basic properties of human vision, mainly, that the human visual system is characterized by a collection of channels that mediate perception. Due to the independent characteristic among the channels, the perception can be predicted channel by channel. In this way, the metric decomposes the original sequence and a distorted version of it into perceptual channels. It then computes a channel-based distortion measure for contrast sensitivity and masking. Throughout our experiments, we have confirmed that MPQM effectively assesses the spatio-temporal video quality degradation by rating the video sequence on a frame by frame basis. However, for the sake of clarity, we report the average MPQM for each videoclip encoding instance.

Figure 5 depicts the video quality using MPQM for different breakpoints applied to the base layer of the Ayersroc,
Hook, and Table Tennis video sequences. As already stated, for the sequences used in our experiments, it was observed that a breakpoint of five could yield a graceful quality degradation (see Figure 6).

From the results obtained in this evaluation, we can make the following observations.

(1) The amount of overhead introduced by the layered video coding scheme is independent of the selected breakpoint, that is, independent of the way the DCT coefficients are split between the two layers. It is clear that due to the need of keeping a perfect synchronization between the two layers, the amount of overhead introduced by the scheme is exclusively due to need of including the syntactic video headers (sequence, GOP, frame, and slice headers) in both video layers and the complete MPEG-2 TS structuring in both layers.

(2) The overhead varies with the $Q$-factor selected. The overhead increases as a function of the $Q$-factor. By increasing the $Q$-factor, the amount of generated semantic data decreases while the amount of generated syntactic data (headers) remains constant.

(3) The traffic distribution between the two layers reaches a saturation point. The value of this saturation point decreases as a function of the $Q$-factor. This is due to the fact that, after the quantization process imbedded in the MPEG-2 video compression algorithm, the number of high-frequency coefficients equal to zero increases as a function of the Qfactor. Since the MPEG-2 video compression algorithm does not encode the DCT coefficients equal to zero, the resulting compressed image will contain a limited number of DCT coefficients. The saturation point represents the point from which all the DCT coefficients have been included in the base layer, leaving the enhancement layer practically empty (except for the overhead introduced by the layering mechanism).

(4) The video quality of the base layer reaches a saturation value. From this saturation point and beyond, the video quality of the base layer remains constant and practically equal to the video quality of the overall image (both layers). 
TABLE 1: Video sequence characteristics.

\begin{tabular}{|c|c|c|c|c|c|}
\hline \multirow{2}{*}{\multicolumn{2}{|c|}{ Video sequence }} & \multicolumn{4}{|c|}{ Video sequences characteristics } \\
\hline & & \multirow{2}{*}{$\frac{\text { Mean bit rate (Kbps) }}{5460}$} & \multirow{2}{*}{$\frac{\text { Frame rate }(\mathrm{fps})}{24}$} & \multirow{2}{*}{$\begin{array}{c}\text { Hdr-bits }(\%) \\
15.08\end{array}$} & \multirow{2}{*}{$\frac{\text { DCT-bits }(\%)}{84.92}$} \\
\hline \multirow{5}{*}{ Ayersroc } & $Q=8$ & & & & \\
\hline & $Q=12$ & 3421 & 24 & 24.64 & 75.36 \\
\hline & $Q=20$ & 1950 & 24 & 40.19 & 59.81 \\
\hline & $Q=28$ & 1455 & 24 & 51.60 & 48.40 \\
\hline & $Q=40$ & 1136 & 24 & 63.42 & 36.58 \\
\hline \multirow{5}{*}{ Hook } & $Q=8$ & 6392 & 24 & 13.40 & 86.6 \\
\hline & $Q=12$ & 3920 & 24 & 22.18 & 77.82 \\
\hline & $Q=20$ & 2272 & 24 & 37.52 & 62.48 \\
\hline & $Q=28$ & 1674 & 24 & 49.46 & 50.54 \\
\hline & $Q=40$ & 1285 & 24 & 62.10 & 37.90 \\
\hline \multirow{5}{*}{ Martin } & $Q=8$ & 4684 & 24 & 15.88 & 84.12 \\
\hline & $Q=12$ & 2670 & 24 & 27.85 & 72.15 \\
\hline & $Q=20$ & 1381 & 24 & 49.44 & 50.56 \\
\hline & $Q=28$ & 983 & 24 & 64.20 & 35.80 \\
\hline & $Q=40$ & 785 & 24 & 75.30 & 24.70 \\
\hline \multirow{5}{*}{ Flower Garden } & $Q=8$ & 14564 & 30 & 8.22 & 91.78 \\
\hline & $Q=12$ & 9672 & 30 & 12.73 & 87.27 \\
\hline & $Q=20$ & 5790 & 30 & 20.01 & 79.99 \\
\hline & $Q=28$ & 4191 & 30 & 27.23 & 72.77 \\
\hline & $Q=40$ & 3043 & 30 & 39.25 & 60.75 \\
\hline \multirow{5}{*}{ Mobile Calendar } & $Q=8$ & 17600 & 30 & 7.31 & 92.69 \\
\hline & $Q=12$ & 11634 & 30 & 11.20 & 88.80 \\
\hline & $Q=20$ & 6906 & 30 & 19.08 & 80.92 \\
\hline & $Q=28$ & 4909 & 30 & 26.92 & 73.08 \\
\hline & $Q=40$ & 3433 & 30 & 38.42 & 61.58 \\
\hline \multirow{5}{*}{ Table Tennis } & $Q=8$ & 12906 & 30 & 11.39 & 88.61 \\
\hline & $Q=12$ & 7972 & 30 & 19.76 & 80.24 \\
\hline & $Q=20$ & 4581 & 30 & 35.51 & 64.49 \\
\hline & $Q=28$ & 3397 & 30 & 45.94 & 54.06 \\
\hline & $Q=40$ & 2581 & 30 & 56.10 & 43.90 \\
\hline \multirow{5}{*}{ Football } & $Q=8$ & 12934 & 30 & 12.14 & 87.86 \\
\hline & $Q=12$ & 8549 & 30 & 18.87 & 81.13 \\
\hline & $Q=20$ & 5169 & 30 & 31.40 & 68.60 \\
\hline & $Q=28$ & 3813 & 30 & 42.28 & 57.72 \\
\hline & $Q=40$ & 2889 & 30 & 54.71 & 45.29 \\
\hline
\end{tabular}

(5) For a breakpoint of 5, a significant amount of video traffic is assigned to the enhancement layer. For instance, for the sequences Ayersroc, Hook, and Table Tennis, an acceptable quality in the base layer can be obtained while the amount of data traffic pertaining to the enhancement layer is in the order of $40 \%, 50 \%$, and $53 \%$, respectively. That is to say, this traffic can be discarded if needed due to a lack of resources (spare network bandwidth) without adversely affecting the overall video service.
The aforementioned analysis sets up the basis to derive the guidelines towards the definition of an adaptive encoding mechanism.

(i) An acceptable breakpoint can be defined as the breakpoint value allowing us to significantly reduce the amount of video data to be included in the base layer for which the quality of the image reconstructed exclusively from the base layer degrades gracefully with respect to the quality of the complete image. 


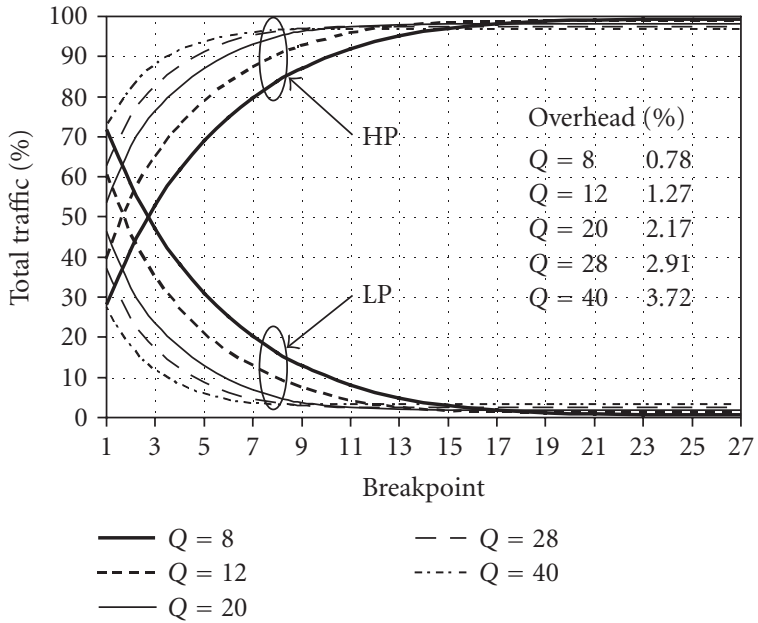

(a)



(b)



(c)

Figure 4: Traffic distribution and overhead versus breakpoint. (a) Ayersroc sequence. (b) Hook sequence. (c) Table Tennis sequence.

TABLE 2: Quality scale.

\begin{tabular}{ccc}
\hline Rating & Impairment & Quality \\
\hline 5 & Imperceptible & Excellent \\
4 & Perceptible, not annoying & Good \\
3 & Slightly & Fair \\
2 & Annoying & Poor \\
1 & Very annoying & Bad \\
\hline
\end{tabular}

(ii) The value of the acceptable breakpoint should be determined taking into account the desired quality of the image and traffic rate pertaining to the base layer.

(iii) The breakpoint and Q-factor have a direct impact on the assignment of the traffic to the enhancement layer and image quality.

\section{A NONLINEAR LAYERED ENCODING SCHEME}

Throughout the previous study, we have defined a constant breakpoint for the whole video sequence. This implies that the layered scheme gives a priority treatment to the B- and P-frames with respect to the I-frames. This is due to the fact that the B-type blocks have a larger number of zeros than the P-type blocks, and these ones contain a larger number of zeros than the I-type blocks. This can in turn be explained by the fact that the B- and P-type blocks contain the prediction errors used in the motion estimation mechanisms. In most cases, the estimation errors are usually small and therefore once quantified, most of them become zero. This is not the case for the I-type blocks, which contain a larger number of nonzero coefficients. This analysis sets the basis towards the definition of a nonlinear layered encoding scheme. In the following, we first review the underlying encoding principles. We then introduce a frame-aware encoding approach based on a nonlinear layered encoding scheme. 


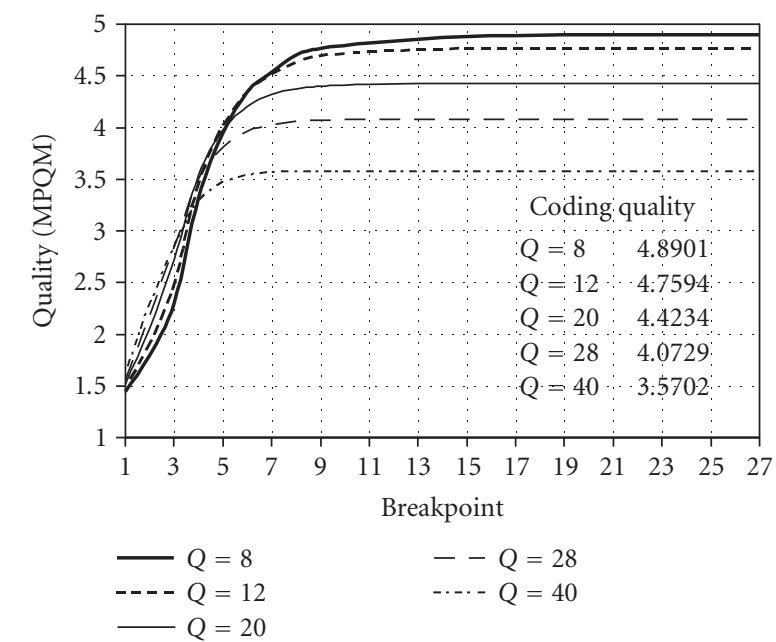

(a)



(b)

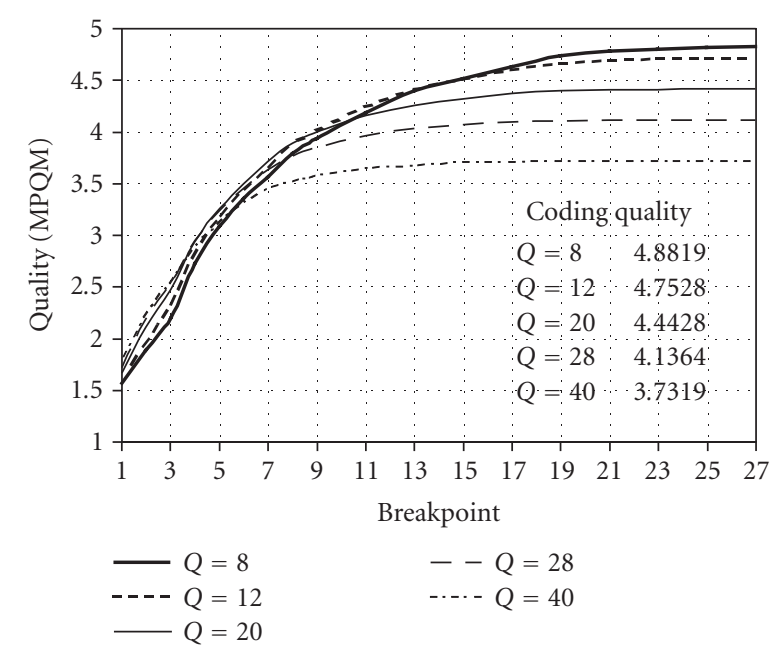

(c)

FIGURE 5: Quantitative video quality for different breakpoints. (a) Ayersroc sequence. (b) Hook sequence. (c) Table Tennis sequence.

\subsection{Encoding principles}

According to the quantization process in most video codecs, the 64 DCT coefficients in a block are scanned in the most efficient manner so that the largest possible zero sequence can be obtained; thus a 64-coefficient block is translated into a few pairs $(u, v)$, where $u$ is the number of DCT coefficients equal to zero before a value $v$ different from zero. These pairs are subsequently coded by means of a codec of variable length, as indicated in Figure 7.

Each pair $(u, v)$ represents a DCT coefficient $v$ different from zero in the block and a number of coefficients $u$ equal to zero in the block. These pairs are the data units that the layering scheme will place in the base layer or in the enhancement layer, in terms of a breakpoint value. The breakpoint defines the number of nonzerovalued DCT coefficients in a block to be included in the base layer.
The fact of defining a single breakpoint value for the whole video sequence means that the same number of pairs $(u, v)$ will be placed in the base layer for the three types of video frames. However, this does not represent the best possible coefficient assignment since not all types of video frames have the same relevance. A natural way of classifying the relevance of the different types of video frames is by understanding their dependencies. Among all types of frames, the Iframes are the most important in the process of reconstructing the video signal. The I-frames contain information that is used in the decoding processes of the two other types of frames, that is, $\mathrm{P}$ - and B-frames. Therefore, an error or loss of information pertaining to an I-frame will have an adverse impact over the decoding processes of the $\mathrm{P}$ - and $\mathrm{B}$-frames depending on it. In a similar way, P-frames are used in the decoding process of B-frames. Therefore, a scheme with a fixed breakpoint does not take into account the unequal relevance of the various types of frames. 




Original image



Breakpoint $=2$

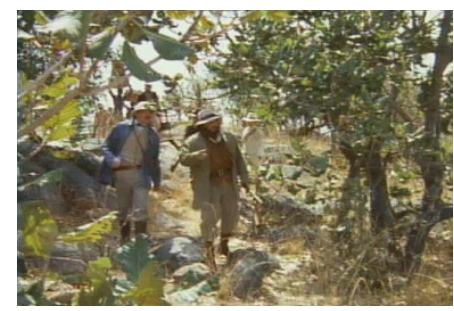

Breakpoint $=5$

(a)

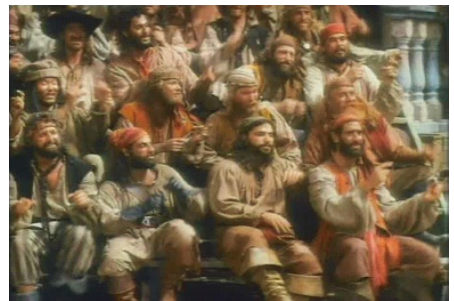

Original image



Breakpoint $=2$



Breakpoint $=5$

(b)

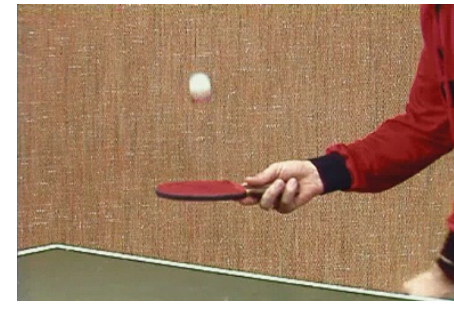

Original image



Breakpoint $=2$

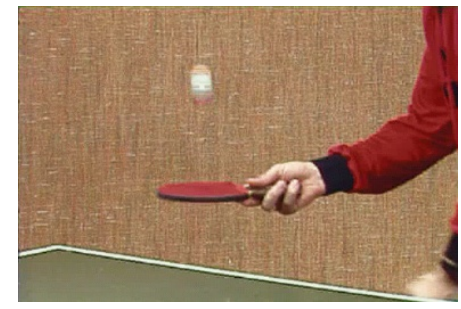

Breakpoint $=5$

(c)

Figure 6: Subjective video quality for different breakpoints. (a) Ayersroc sequence (I-picture). (b) Hook sequence (P-picture). (c) Table Tennis sequence (B-picture).

\subsection{A frame-aware encoding approach}

In order to take into account the relevance of the various types of frames, several proposals studies have suggested the use of a different breakpoint for each type of frame. This can be done by defining breakpoint corrective factors, taking values between 0 and 1 , to be used for P- and B-frames. Once a breakpoint has been assigned to I-frames, a reduced breakpoint is used to encode P-type blocks and an even lower corrective factor is assigned for the encoding process of B-type blocks. Even though some studies have shown the benefits of using this scheme in terms of the quality of the video signals reconstructed by exclusively using the base layer, the number of cases evaluated has been rather limited. Furthermore, little attention has been paid to study the effect of varying the breakpoint factor, another major video coding parameter affecting the quality of the video signal as well as the data generation rate.

In the following, we carried an exhaustive study by applying a frame-aware nonlinear encoding scheme to seven different video sequences, each one encoded using six different sets of breakpoint values and four different $Q$-factor values.
The nonlinear video encoding scheme is evaluated in terms of the quality of the decoded base layer and its corresponding data rate.

\subsection{Numerical results}

In this section, we will evaluate the frame-aware nonlinear encoding scheme. In the previous section, we have found out that the breakpoint of five provides graceful quantitative and subjective (visual) video qualities degradation for most of the video sequences. Table 3 shows the corrective factors used in our studies. As seen from the table, the first set of values corresponds to the assignment of the same breakpoint value to all frames. For the other five cases, we have applied a corrective value to reduce the number of DCT coefficients pertaining to the $\mathrm{P}$ - and $\mathrm{B}$-frames.

Figures 8,9 , and 10 , show the performance of the scheme under study for the video sequences Ayersroc, Hook, and Table Tennis encoded using a $Q$-factor set to 8 and 12. The figure shows the mean number of DCT coefficients in each type of frame, the percentage of traffic corresponding to the enhancement layer, and the quality of the video sequence decoded using only the base layer. 

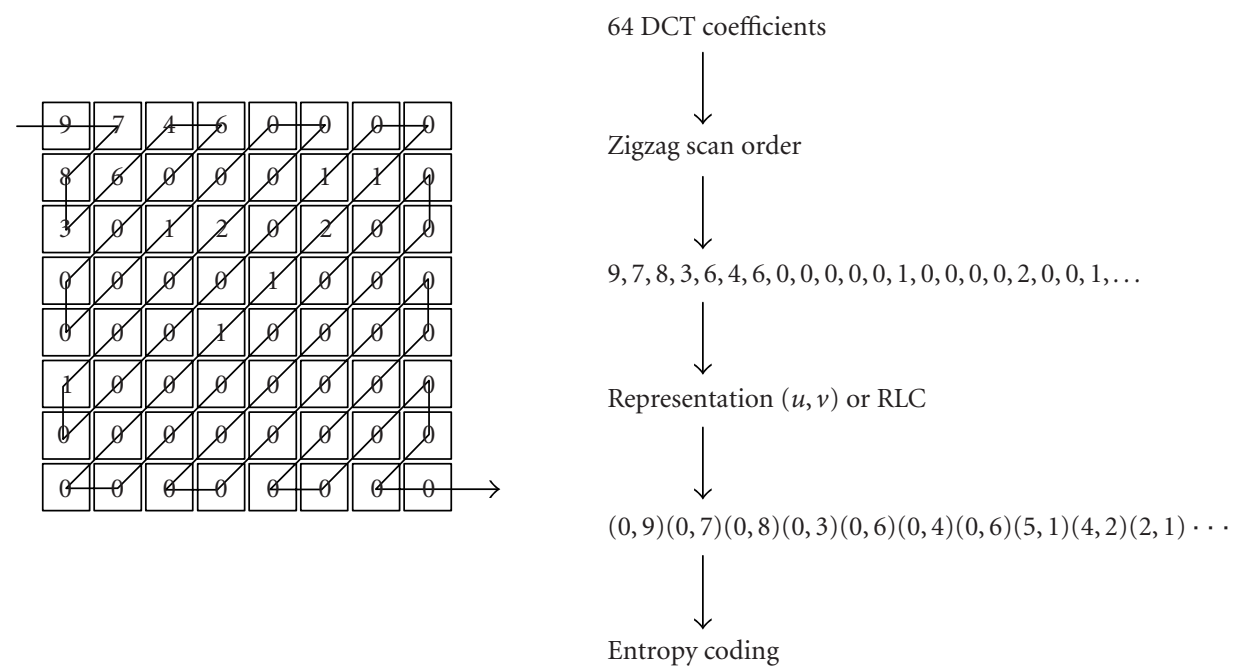

FIgURE 7: A 64-DCT block representation in pairs $(u, v)$.

TABLE 3: Corrective factors.

\begin{tabular}{clll}
\hline Option & $\mathrm{I}$ & $\mathrm{P}$ & $\mathrm{B}$ \\
\hline Uniform (static) & 1 & 1 & 1 \\
O1 & 1 & 0.75 & 0.75 \\
O2 & 1 & 0.75 & 0.5 \\
O3 & 1 & 0.75 & 0.25 \\
O4 & 1 & 0.5 & 0.5 \\
O5 & 1 & 0.5 & 0.25 \\
\hline
\end{tabular}

From the figures, it is clear that for each breakpoint value, a uniform breakpoint assignment results in the best video quality. However, this set-up corresponds to the lowest percentage of traffic assigned to the enhancement layer (highest percentage of traffic assigned to the base layer). From the results, it is clear that the quality of the video reconstructed using exclusively the base layer deteriorates as the size of the enhancement layer increases (more data is removed from the base layer). It is clear from the figure that in order to improve the overall system performance in terms of the quality and traffic volume, it is necessary not only to apply a corrective factor but as well to change the breakpoint value. From Figures 8-10, we observe that when the breakpoint value is set to 8 and by using a corrective factor option, such as $\mathrm{O} 3$ or $\mathrm{O} 5$, we are able to move an important amount of data to the enhancement layer and obtain a better or comparable video quality to the one achieved using a uniform breakpoint assignment and a breakpoint $=5$.

A closer look at Figures 8-10 also shows that by making the aforementioned changes to the encoding parameters, the system performance has been improved by the fact of including more I-frame DCT coefficients into the base layer. More specifically, the nonlinear scheme provides a better overall assignment of the DCT coefficients in a block to the base layer when compared to the linear encoding scheme. The figures clearly show the trend of the proposed scheme. By taking into account the relevance of the different frame types, the nonlinear scheme is able to include a larger number of DCT coefficients pertaining to the I-frames while reducing accordingly the number of DCT coefficients belonging to the P- and $\mathrm{B}$-frames. It is also worth to mention that this trend is accentuated as the breakpoint is increased from 5 to 8 . This translates into a better or similar image quality of the base layer as well as an important decrease in the base layer traffic, that is, a reduction in the amount of the data required to achieve an acceptable video quality. The best results are obtained when applying options $\mathrm{O} 3$ and $\mathrm{O} 5$, $\mathrm{O} 5$ being the most restrictive of the policies under study; only half and a quarter of the DCT coefficients belonging to the P- and $\mathrm{B}$-frame types are included in the base layer, respectively. These results clearly show the effectiveness of the proposed scheme.

In order to further explore the performance of this encoding scheme, we have evaluated seven different video sequences, encoded using four different values for the $Q$-factor as well as the six corrective breakpoint assignments. Table 4 lists the results obtained when using the uniform assignment case and option $\mathrm{O} 5$.

A closer look at the results in Table 4 allows us to make the following observations.

(i) In all cases, there is a significant increase in the amount of data being moved to the enhancement layer. The change is far more important for low values of the $Q$ factor.

(ii) For all but one sequence, the quality of the base layer is improved when using option $\mathrm{O} 5$ and breakpoint $=8$ as compared to the case breakpoint $=5$ and a uniform breakpoint value.

(iii) The fact that the video quality of the base layer is gracefully degraded by the use of the different proposed 


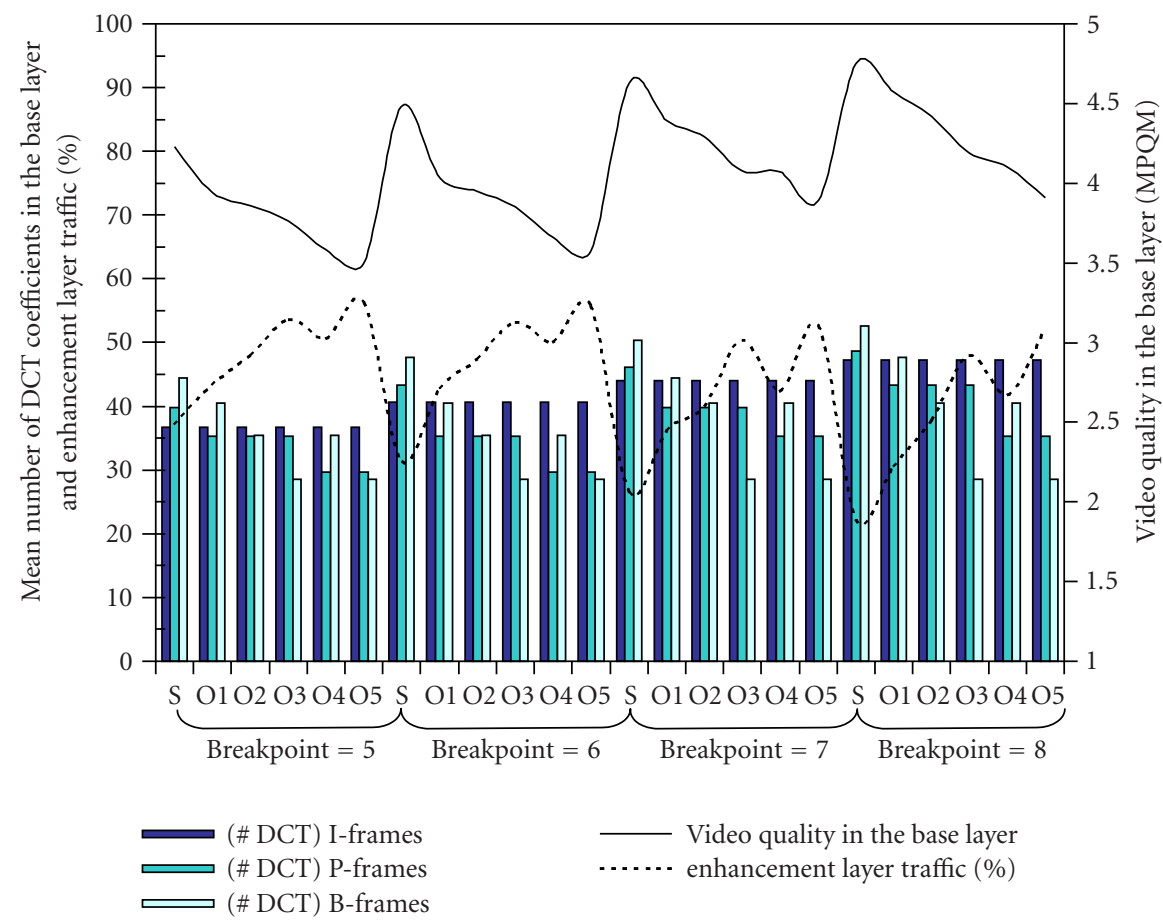

(a)

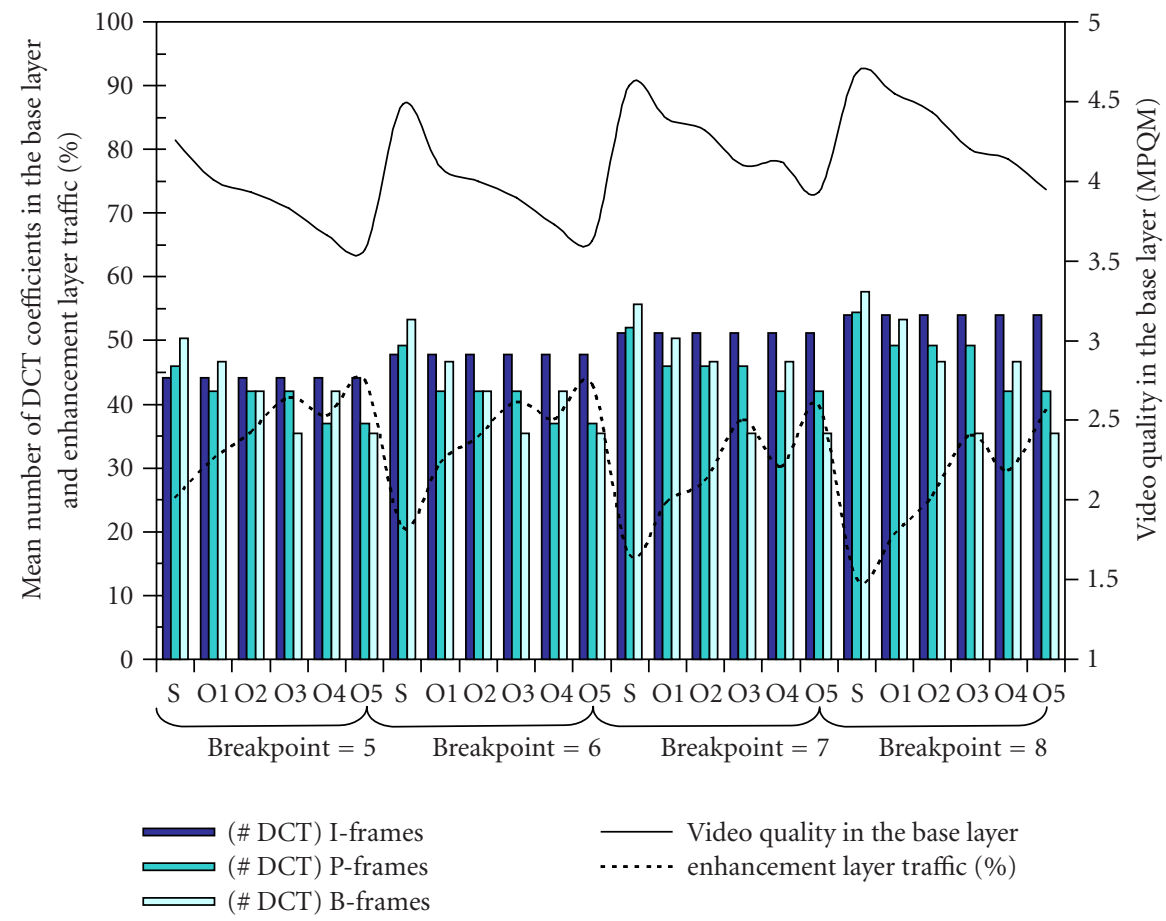

(b)

Figure 8: Performance of the frame-aware layering scheme for the Hook video sequence: (a) $Q=8$ and (b) $Q=12$.

options 1 to 5 clearly shows the benefits of taking into account the relevance of the different pieces of information. As we increase the breakpoint value while using option $\mathrm{O} 5$, we are placing a greater number of DCT coefficients pertaining to the I-frames into the base layer. Since the P- and B-frames within 


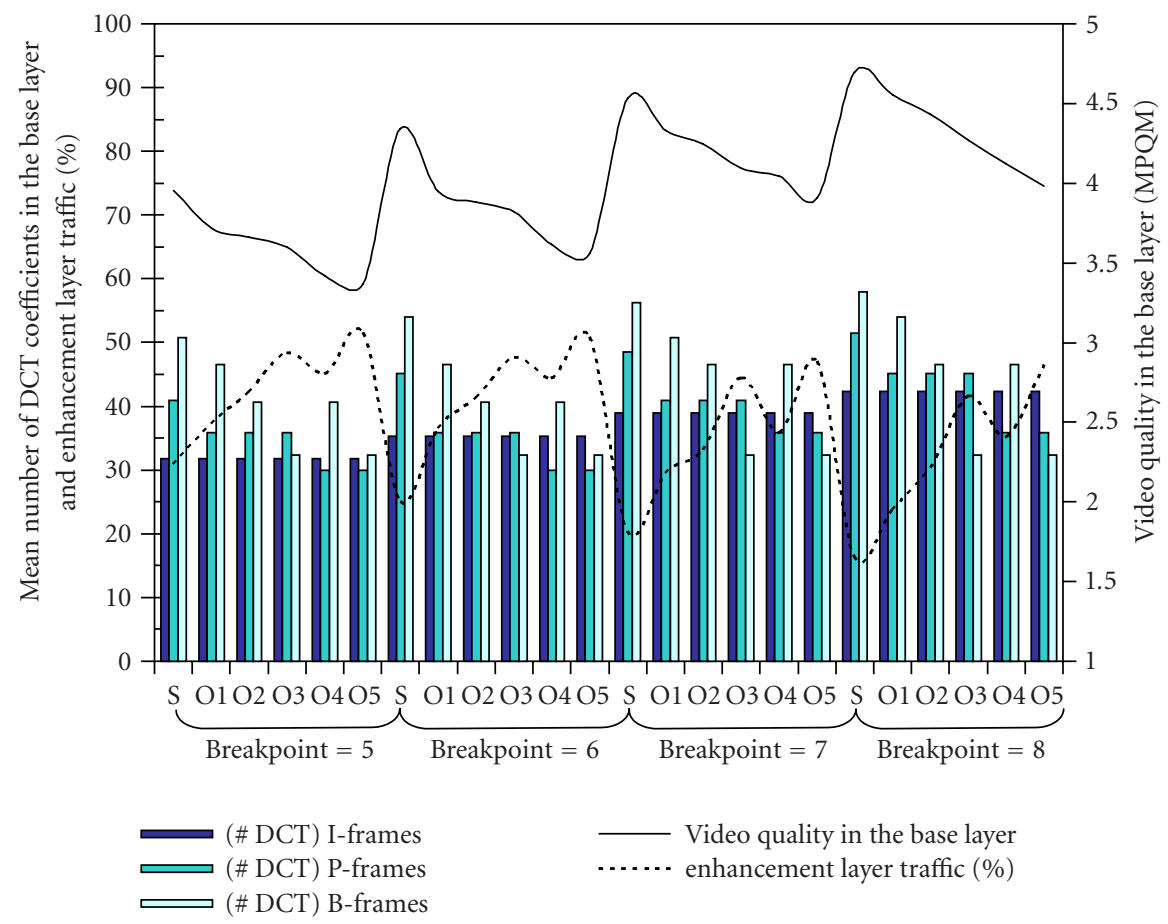

(a)



(b)

FIGURE 9: Performance of the frame-aware layering scheme for the Ayersroc video sequence: (a) $Q=8$ and (b) $Q=12$.

a GOP will be decoded using as reference the I-frame, the increase of the quality in the I-frames paysoff. The inclusion of more DCT coefficients belonging to the I-frames compensates the reduction of video quality of the base layer assigned to the P- and Bframes. 




(a)



(b)

FIgURE 10: Performance of the frame-aware layering scheme for the Table Tennis video sequence: (a) $Q=8$ and (b) $Q=12$.

From the results, it is clear that the DCT-based nonlinear layered video codec shows a good compromise between the amount of relevant traffic and the quality of the image.
Our results set the basis towards the definition of the major guidelines to configure the encoding parameters: breakpoint, corrective factors, and $Q$-factor. The major guidelines can be 
TABLE 4: Performance results.

\begin{tabular}{|c|c|c|c|c|c|c|c|}
\hline \multirow[b]{2}{*}{ Video sequence } & & \multicolumn{3}{|c|}{ Uniform option (breakpoint $=5$ ) } & \multicolumn{3}{|c|}{ Frame-aware option O5 (breakpoint = 8) } \\
\hline & & $\begin{array}{c}\text { DCT No. of } \\
\text { coefficients for I, P, B }\end{array}$ & LP traffic $(\%)$ & HP quality & $\begin{array}{c}\text { DCT No. of } \\
\text { coefficients for I, P, B }\end{array}$ & LP traffic $(\%)$ & HP quality \\
\hline \multirow{4}{*}{ Ayersroc } & $Q=8$ & $31.70,40.94,50.84$ & 30.90 & 3.95 & $42.37,35.93,32.30$ & 46.41 & 3.98 \\
\hline & $Q=12$ & $38.23,49.09,56.15$ & 20.98 & 4.01 & $49.72,44.69,41.94$ & 31.90 & 4.02 \\
\hline & $Q=20$ & $47.71,55.40,58.78$ & 12.85 & 3.97 & $58.29,51.57,47.29$ & 18.76 & 3.95 \\
\hline & $Q=40$ & $59.29,61.41,62.52$ & 5.89 & 3.46 & $63.52,59.07,52.14$ & 7.95 & 3.44 \\
\hline \multirow{4}{*}{ Hook } & $Q=8$ & $36.65,39.81,44.47$ & 37.14 & 4.23 & $47.22,35.36,28.52$ & 52.18 & 3.91 \\
\hline & $Q=12$ & $44.14,46.02,50.37$ & 25.28 & 4.26 & $50.03,42.07,35.44$ & 39.27 & 3.95 \\
\hline & $Q=20$ & $53.09,52.47,56.03$ & 13.94 & 4.17 & $60.67,48.40,40.41$ & 24.23 & 3.91 \\
\hline & $Q=40$ & $61.75,61.48,62.70$ & 4.72 & 3.53 & $63.88,58.85,50.96$ & 8.64 & 3.44 \\
\hline \multirow{4}{*}{ Martin } & $Q=8$ & $35.83,48.29,54.00$ & 24.90 & 4.51 & $46.35,43.26,34.92$ & 41.19 & 4.60 \\
\hline & $Q=12$ & $44.88,56.51,59.65$ & 14.97 & 4.47 & $54.90,53.49,47.36$ & 23.91 & 4.54 \\
\hline & $Q=20$ & $56.40,60.55,62.04$ & 8.69 & 4.27 & $61.89,58.65,54.62$ & 11.94 & 4.30 \\
\hline & $Q=40$ & $62.91,63.30,63.53$ & 5.51 & 3.69 & $63.87,62.67,60.00$ & 6.22 & 3.69 \\
\hline \multirow{4}{*}{ Flower Garden } & $Q=8$ & $23.36,25.67,34.49$ & 58.42 & 2.20 & $29.42,22.61,22.04$ & 68.93 & 2.50 \\
\hline & $Q=12$ & $27.35,31.15,43.59$ & 46.27 & 2.30 & $34.25,27.67,28.47$ & 57.78 & 2.60 \\
\hline & $Q=20$ & $34.01,40.26,54.56$ & 30.25 & 2.47 & $41.05,36.06,39.39$ & 39.74 & 2.80 \\
\hline & $Q=40$ & $44.73,55.13,62.16$ & 12.95 & 2.64 & $52.49,51.49,53.48$ & 15.39 & 3.01 \\
\hline \multirow{4}{*}{ Mobile Calendar } & $Q=8$ & $20.29,30.96,39.35$ & 62.22 & 1.95 & $27.80,27.66,27.48$ & 71.81 & 2.68 \\
\hline & $Q=12$ & $24.92,36.96,44.89$ & 51.97 & 2.05 & $33.23,33.49,32.86$ & 62.28 & 2.75 \\
\hline & $Q=20$ & $32.19,45.00,51.68$ & 36.41 & 2.19 & $41.61,41.41,39.50$ & 46.48 & 2.84 \\
\hline & $Q=40$ & $44.65,55.57,58.92$ & 16.83 & 2.32 & $52.50,52.58,49.57$ & 21.46 & 2.82 \\
\hline \multirow{4}{*}{ Table Tennis } & $Q=8$ & $32.07,38.75,46.49$ & 52.06 & 3.10 & $40.85,35.34,33.83$ & 63.04 & 3.45 \\
\hline & $Q=12$ & $40.46,44.56,51.60$ & 42.65 & 3.18 & $48.52,41.75,41.28$ & 51.93 & 3.54 \\
\hline & $Q=20$ & $49.28,48.85,55.99$ & 28.95 & 3.27 & $54.46,45.89,45.75$ & 37.64 & 3.61 \\
\hline & $Q=40$ & $57.09,58.51,61.44$ & 10.72 & 3.12 & $60.25,56.34,54.12$ & 14.28 & 3.37 \\
\hline \multirow{4}{*}{ Football } & $Q=8$ & $25.19,30.52,38.61$ & 49.19 & 2.31 & $34.16,26.46,23.93$ & 58.74 & 2.32 \\
\hline & $Q=12$ & $31.91,38.14,43.47$ & 39.15 & 2.40 & $44.04,34.20,29.91$ & 48.93 & 2.43 \\
\hline & $Q=20$ & $45.51,45.58,48.51$ & 25.70 & 2.57 & $55.75,41.71,33.73$ & 34.48 & 2.70 \\
\hline & $Q=40$ & $58.23,54.76,57.89$ & 9.66 & 2.73 & $62.08,51.3,42.77$ & 15.05 & 2.78 \\
\hline
\end{tabular}

simply stated as follows:

(i) for a target video quality, set $Q$ to a given (constant) value;

(ii) determine a breakpoint ensuring a minimum acceptable video quality;

(iii) using as a basis the breakpoint in the previous point, apply a corrective factor to the breakpoint to be used to encode the $\mathrm{P}$ - and $\mathrm{B}$-frames;

(iv) the factor to apply to the P-frames should be larger than the factor to be used for the B-frames.

A video encoder incorporating these features could adapt its encoding parameters in response to a congestion signal from the network in order to reduce the amount of data while maintaining the best possible acceptable video quality. For instance, assume that under normal operation, the encoder operates with a breakpoint $=5$ and a uniform corrective factor; in response to a congestion condition, the encoder could change both the breakpoint and the corrective factors. If a more severe congestion condition is encountered, the encoder could decide to change only the corrective factors that for all cases results in a significant video-generation rate drop. This latter mode of operation proves to be very useful due to the fact that under severe network conditions, the encoder can quickly react by simply changing the corrective factors without having to change the $Q$-factor.

\section{CONCLUSIONS}

In this paper, we have analyzed the performance of a video encoding scheme able to improve the video quality while reducing the amount of data used to represent the video 
sequence. We have analyzed the scheme by applying it to seven different video sequences and by changing the encoder parameters. From our results, we have found that, for most of the cases analyzed, it is feasible to gracefully degrade or even ensure a minimum acceptable video quality while reducing the amount of data used to represent the video sequence. We have therefore derived the major guidelines to set up the encoding parameters. We have also outlined an adaptive encoding process to be used in conjunction with a rate-based congestion control mechanism.

\section{ACKNOWLEDGMENTS}

This work was supported by the Ministry of Science and Technology of Spain under CICYT Project TIC2003-08154C06-02, the Council of Science and Technology of Castilla-La Mancha under Project PBC-03-001, and the Natural Sciences and Engineering Council of Canada.

\section{REFERENCES}

[1] P. Cuenca, L. Orozco-Barbosa, F. Quiles, and A. Garrido, "Loss-resilient ATM protocol architecture for MPEG-2 video communications," IEEE Journal on Selected Areas in Communications, vol. 18, no. 6, pp. 1075-1086, 2000.

[2] P. Cuenca, A. Garrido, F. Quiles, and L. Orozco-Barbosa, "Some proposals to improve error resilience in the MPEG-2 video transmission over ATM networks," in Proc. 17th Annual Joint Conference of the IEEE Computer and Communications Societies, vol. 2, pp. 668-675, San Francisco, Calif, USA, March-April 1998.

[3] P. Cuenca, A. Garrido, F. Quiles, and L. Orozco-Barbosa, "An efficient protocol architecture for error-resilient MPEG-2 video communications over ATM networks," IEEE Transactions on Broadcasting, vol. 45, no. 1, pp. 129-140, 1999.

[4] ISO/IEC 14496, Information technology-Generic coding of audio-visual objects- Part 2: Visual. Final Committee Draft of International Standard, Seoul, March 1999. Part 1: System; Part 2: Visual; Part 3: Audio, Part 4: Conformance Testing; Part 5: Technical Report; Part 6: Delivery Multimedia Integration Framework.

[5] ISO/IEC 13818-1, "Information technology-generic coding of moving pictures and associated audio informationPart 1: Systems," International Standard, ISO/IEC/JTC1/SC29 WG11, 2000.

[6] ISO/IEC 13818-2, "Information technology—generic coding of moving pictures and associated audio information-Part 2: Video," International Standard, ISO/IEC/JTC1/SC29 WG11, 2000.

[7] ISO/IEC 13818-3, "Information technology—generic coding of moving pictures and associated audio information-Part 3: Audio," International Standard, ISO/IEC/JTC1/SC29 WG11, 1998.

[8] ISO/IEC 11172-1, "Information technology_coding of moving pictures and associated audio for digital storage media at up to about 1,5 mbits-Part 1: Systems," International Standard, ISO/IEC/JTC1/SC29 WG11, 1993.

[9] ISO/IEC 11172-2, "Information technology-coding of moving pictures and associated audio for digital storage media at up to about 1,5 mbits-Part 2: Video," International Standard, ISO/IEC/JTC1/SC29 WG11, 1993.

[10] ISO/IEC 11172-3, "Information technology_coding of moving pictures and associated audio for digital storage media at up to about 1,5 mbits-Part 3: Audio," International Standard, ISO/IEC/JTC1/SC29 WG11, 1993.
[11] ISO/IEC 11172-4, "Information technology-coding of moving pictures and associated audio for digital storage media at up to about 1,5 mbits-Part 4: Compliance testing," International Standard, ISO/IEC/JTC1/SC29 WG11, 1995.

[12] ISO/IEC 14496-1, "Information technology-coding of audio-visual objects-Part 1: Systems," International Standard, ISO/IEC/JTC1/SC29 WG11, 2001.

[13] ISO/IEC 14496-2, "Information technology-coding of audio-visual objects_-Part 2: Visual," International Standard, ISO/IEC/JTC1/SC29 WG11, 2004.

[14] ISO/IEC 14496-3, "Information technology_coding of audio-visual objects-Part 3: Audio," International Standard, ISO/IEC/JTC1/SC29 WG11, 2001.

[15] ISO/IEC 14496-4, "Information technology_coding of audio-visual objects-Part 4: Conformance testing," International Standard, ISO/IEC/JTC1/SC29 WG11, 2000.

[16] ISO/IEC 14496-5, "Information technology-coding of audio-visual objects-Part 5: Reference software," International Standard, ISO/IEC/JTC1/SC29 WG11, 2001.

[17] ISO/IEC 14496-6, "Information technology-coding of audio-visual objects-Part 6: Delivery multimedia integration framework (dmif)," International Standard, ISO/IEC/JTC1/SC29 WG11, 2000.

[18] S. Worrall, S. Fabri, A. H. Sadka, and A. M. Kondoz, "Prioritisation of data partitioned MPEG-4 video over mobile networks," ETT-European Transactions on Telecommunications, vol. 12, no. 3, pp. 169-174, 2001.

[19] M. Ghanbari, "Two-layer coding of video signals for VBR networks," IEEE Journal on Selected Areas in Communications, vol. 7, no. 5, pp. 771-781, 1989.

[20] P. Pancha and M. El Zarki, "MPEG coding for variable bit rate video transmission," IEEE Communications Magazine, vol. 32, no. 5, pp. 54-66, 1994.

[21] M. R. Ismail, I. E. Lambadaris, M. Devetsikiotis, and A. R. Kaye, "Modelling prioritized MPEG video using TES and a frame spreading strategy for transmission in ATM networks," in Proc. 14th Annual Joint Conference of the IEEE Computer and Communications Societies, vol. 2, pp. 762-770, Boston, Mass, USA, April 1995.

[22] M. R. Karim, ATM Technology and Services Delivery, Prentice Hall, Upper Saddle River, NJ, USA, 2000.

[23] ETSI, Broadband Radio Access Networks (BRAN) HIPERLAN type 2-Data link control (DLC)_Part 1: Basic data transport functions, TS 101 761-1, December 2001.

[24] IEEE 802.11a/D5.0, Wireless LAN medium access control (MAC) and physical layer (PHY) specifications: higher speed physical layer (PHY) extension in the $5 \mathrm{GHz}$ band, April 1999.

[25] IEEE 802.11b/D5.0, Wireless LAN medium access control (MAC) and physical layer (PHY) specifications: higher speed physical layer (PHY) extension in the $2.4 \mathrm{GHz}$ band, April 1999.

[26] M. Toukourou and L. Orozco-Barbosa, "On the performance of MPEG-2 video-on-demand over RSVP," in Proc. SPIE Network Control and Service Quality Conference, vol. 4211 of Proceedings of SPIE, pp. 13-24, Boston, Mass, USA, November 2000.

[27] E. Rosen, A. Viswanathan, and R. Callon, "Multiprotocol label switching architecture,” RFC 3031, January 2001.

[28] H. Yu, D. Makrakis, and L. Orozco-Barbosa, "Experimental evaluation of MPEG-2 video over differentiated services IP networks," in Proc. IEEE Pacific Rim Conference on Communications, Computers and Signal Processing, vol. 2, pp. 469-472, Victoria, BC, Canada, August 2001.

[29] K. N. Ngan, C. W. Yap, and K. T. Tan, Video Coding for Wireless Communication Systems, Signal Processing and Communication Series. Marcel Dekker, New York, NY, USA, 2001. 
[30] L. Hanzo, P. J. Cherriman, and J. Streit, Wireless Video Communications: Second to Third Generation Systems and Beyond, Mobile \& Wireless Communications. Wiley-IEEE Computer Society, New York, NY, USA, 2001.

[31] C. J. Van den Branden and O. Verscheure, "Perceptual quality measure using a spatio-temporal model of the human visual system," in Proc. SPIE Digital Video Compression: Algorithms and Technologies, vol. 2668 of Proceedings of SPIE, pp. 450 461, San Jose, Calif, USA, January 1996.

[32] P. Frossard and O. Verscheure, "AMISP: a complete contentbased MPEG-2 error-resilient scheme," IEEE Trans. Circuits and Systems for Video Technology, vol. 11, no. 9, pp. 989-998, 2001.

[33] O. Verscheure, P. Frossard, and M. Hamdi, "User-oriented QoS analysis in MPEG-2 video delivery," Journal of Real-Time Imaging, vol. 5, no. 5, pp. 305-314, 1999.

Pedro Cuenca received his M.S. degree in physics (electronics and computer science, extraordinary award) from the University of València in 1994. He got his Ph.D. degree in computer engineering in 1999 from the Polytechnic University of Valencia, Spain. In 1995, he joined the Department of Computer Engineering, the University of Castilla-La Mancha. He is currently an Associate Professor of communications

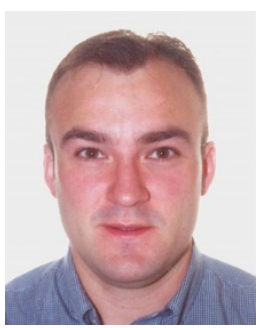
and computer networks. He has also been a Visiting Researcher at the Nottingham Trent University, Nottingham, England, and at the Multimedia Communications Research Laboratory, University of Ottawa, Canada. His research topics are centered in the area of high-performance networks, wireless LAN, video compression, QoS video transmission, and error-resilient protocol architectures. He has served in the organization of international conferences as a Session Chair. He has been a reviewer for several journals and for several international conferences. He is a member of the IFIP 6.8 Working Group and a member of the IEEE.

Luis Orozco-Barbosa received his B.S. degree in electrical and computer engineering from Universidad Autónoma Metropolitana, Mexico, in 1979, the Diplome d'études Approfondies from École Nationale Supérieure d'Informatique et de Mathématiques Appliquées (ENSIMAG), France, in 1984, and the Doctorat de l'Université from Université Pierre et Marie Curie, France, in 1987, both in computer

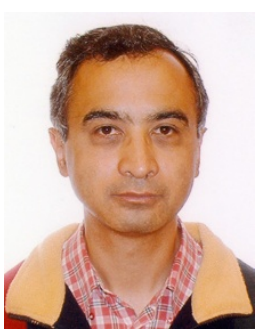
science. From 1991 to 2002, he was a faculty member in the School of Information Technology and Engineering (SITE), University of Ottawa, Canada. In 2002, he joined the Department of Computer Engineering at the University of Castilla-La Mancha, Spain. He has also been appointed a Director of the Albacete Research Institute of Informatics, a National Centre of Excellence. He has conducted numerous research projects with the private sector and served as a Technical Advisor for the Canadian International Development Agency (CIDA). He has published over 180 papers in international journals and conferences on computer networks and performance evaluation. His current research interests include Internet protocols, network planning, wireless communications, traffic modeling, and performance evaluation. He is a member of the IEEE.
Francisco Delicado received his M.S. degree in physics (electronics and computer science) from the University Complutense of Madrid, Madrid, Spain, in 1995. He is currently a Ph.D. degree student in the Department of Computer Engineering at the University of Castilla-La Mancha. His research interests include high-performance networks, wireless networks, video compression, video transmission, and errorresilient protocol architectures.

Antonio Garrido received the degree in physics (electronics and computer science) and the Ph.D. degrees from the University of Granada, Spain, in 1986 and University of Valencia, Spain, in 1991, respectively. In 1986, he joined the Department of Computer Engineering at the University of Castilla-La Mancha, where he is currently a Full Professor of Computer Architecture and Technology and Dean of the Es-

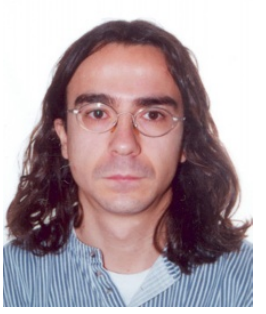
cuela Politécnica Superior de Albacete (School of Computer Engineering). His research interests include high-performance networks, telemedicine, video compression, and video transmission. He has published over 40 papers in international journals and conferences on performance evaluation of parallel computer and communications systems and video compression and transmission in high-speed networks. He has led several research projects in telemedicine, computer networks and advanced computer system architectures. 